\title{
Extreme X-ray Behavior in the Low-Luminosity Active Nucleus of NGC 4395
}

\author{
Edward C. Moran, ${ }^{1,2}$ Michael Eracleous, ${ }^{3}$ Karen M. Leighly, ${ }^{4}$ George Chartas, ${ }^{3}$ Alexei V. \\ Filippenko, ${ }^{2}$ Luis C. Ho, ${ }^{5}$ and Philip R. Blanco ${ }^{6}$
}

\begin{abstract}
We present the results of a 17 ks Chandra observation of the nearby dwarf spiral galaxy NGC 4395, focusing on the X-ray properties of the moderate-mass black hole that resides in its nucleus. Chandra affords the first high-quality, broadband X-ray detection of this object that is free of contamination from nearby sources in the field. We find that the nuclear X-ray emission is unresolved in the Chandra image and confirm the rapid, large-amplitude X-ray variability reported in previous studies. The spectrum of the nuclear source shows evidence for absorption by an ionized medium. There is also evidence for spectral variability over the course of the Chandra observation, although contrary to prior reports, it appears to be uncorrelated with fluctuations in the hard $\mathrm{X}$-ray count rate. It is possible that the short-term spectral variability results from column density changes in the ionized absorber. By far the most unusual high-energy property of NGC 4395 is the shape of its spectrum above $1 \mathrm{keV}$. The Chandra data indicate a power-law photon index of $\Gamma \approx 0.6$, which is much flatter than the $\Gamma \approx 1.8 \mathrm{X}$ ray spectra typical of active galactic nuclei and the slope of the nuclear X-ray spectrum measured from an earlier ASCA observation of NGC 4395. This extreme flatness and dramatic long-term variability of the X-ray spectrum are unprecedented among active galactic nuclei. A variety of possibilities for the origin of the flat continuum slope are considered; none provides a fully satisfactory explanation.
\end{abstract}

Subject headings: galaxies: individual (NGC 4395) — galaxies: Seyfert — X-rays: galaxies

\footnotetext{
${ }^{1}$ Chandra Fellow.

${ }^{2}$ Department of Astronomy, University of California, 601 Campbell Hall, Berkeley, CA 94720-3411.

${ }^{3}$ Department of Astronomy and Astrophysics, The Pennsylvania State University, 525 Davey Laboratory, University Park, PA 16802.

${ }^{4}$ Department of Physics and Astronomy, The University of Oklahoma, 440 West Brooks Street, Norman, OK 73019 .

${ }^{5}$ The Observatories of the Carnegie Institution of Washington, 813 Santa Barbara Street, Pasadena, CA 911011292.

${ }^{6}$ Center for Astrophysics and Space Sciences, University of California, San Diego, 9500 Gilman Drive, La Jolla, CA 92093-0111.
} 


\section{Introduction}

The optical/ultraviolet spectrum of the nucleus of NGC 4395, a nearby dwarf spiral galaxy, exhibits many of the features that characterize the spectra of luminous type 1 Seyfert galaxies and quasars (Filippenko \& Sargent 1989; Filippenko, Ho, \& Sargent 1993). In addition to a featureless continuum and strong forbidden emission lines, some of which indicate a very high degree of ionization, NGC 4395 displays broad permitted emission lines with velocity widths of several thousand $\mathrm{km} \mathrm{s}^{-1}$. Additional evidence from across the electromagnetic spectrum (Moran et al. 1999; Lira et al. 1999; Kraemer et al. 1999; Iwasawa et al. 2000; Wrobel, Fassnacht, \& Ho 2001) supports the hypothesis that NGC 4395 is powered by accretion onto a black hole, similar to other active galactic nuclei (AGNs).

In a number of respects, however, the AGN in NGC 4395 is a prominent outlier. Most notably, with an absolute $B$ magnitude of -11 and an $\mathrm{H} \alpha$ luminosity of a few times $10^{38} \mathrm{ergs} \mathrm{s}^{-1}$, it is the optically least luminous broad-line AGN currently known. Based on stellar velocity dispersion measurements and other arguments (Filippenko \& Ho 2002), the mass of the black hole in NGC 4395 is constrained to be at most $\sim 8 \times 10^{5} M_{\odot}$, and probably less than $\sim 10^{5} M_{\odot}-$ significantly smaller than the black holes found in the nuclei of other galaxies, both active and quiescent. Possibly related to its low black-hole mass, the AGN in NGC 4395 is located in a nearly bulgeless dwarf galaxy; in contrast, other galaxies known to contain nuclear black holes are more massive and have well-developed bulges. NGC 4395 displays some exceptional emission characteristics as well. For example, it is one of the most X-ray-variable AGNs known (Iwasawa et al. 2000), and its radio-to-X-ray spectral energy distribution differs markedly from those of other types of AGNs, perhaps indicating that there is something special about the accretion processes in NGC 4395. $\mathrm{X}$-ray emission is directly linked to the accretion processes in AGNs. In this paper, we present the results of a high spatial resolution, broadband Chandra observation of the central X-ray source in NGC 4395, providing new insights into the nuclear activity in this unusual object. We adopt a distance of $4.1 \mathrm{Mpc}$ for NGC 4395, based on Hubble Space Telescope observations of the brightest asymptotic giant branch stars in the galaxy (Minitti et al. 2002).

\section{The Chandra Observation}

NGC 4395 has been the target of pointed X-ray observations in the $0.1-2.4 \mathrm{keV}$ band with ROSAT (Moran et al. 1999; Lira et al. 1999) and in the 0.6-10 keV band with ASCA (Iwasawa et al. 2000). However, because of the modest spectral coverage and collecting area of ROSAT and the poor angular resolution of ASCA, a complete picture of the high-energy properties of NGC 4395 is lacking. Chandra provides the first broadband, high-resolution X-ray view of this object.

NGC 4395 was observed with Chandra on 20 June 2000 (UT) for 17,187 s with the ACIS-S instrument. The S3 chip was the only active CCD; a $1 / 2$ subarray was read out to reduce the possibility of photon pile-up, which yielded a frame time of $1.54 \mathrm{~s}$. The nucleus of the galaxy was 
located at the instrument aim point. We reprocessed the data with the CIAO software, version 2.1.2, in order to remove the 0.5 pixel randomization introduced during the standard processing of the data. The screened data consist of events with grades $0,2,3,4$, and 6; no background flares occurred during the observation. In the following sections, we describe the X-ray imaging, variability, and spectroscopy results obtained with Chandra.

\section{X-ray Imaging}

The ROSAT PSPC image of NGC 4395 revealed that there are at least five X-ray sources within the central 2!8 of the galaxy (Moran et al. 1999), one of which is significantly brighter than the nucleus at low X-ray energies. Thus, in the images obtained with ASCA (half-power diameter $\approx 3^{\prime}$ ), the nuclear source was significantly contaminated by emission from the other nearby sources, particularly below $2 \mathrm{keV}$ (Iwasawa et al. 2000). As Figure 1 illustrates, the nucleus (source A) is completely isolated in the $\sim 1^{\prime \prime}$ resolution Chandra image. ${ }^{7}$ The high angular resolution of Chandra also allows us to measure the position and spatial extent of the nuclear X-ray emission in NGC 4395 with unprecedented accuracy. The centroid of the nuclear source is $\alpha(2000)=12^{\mathrm{h}} 25^{\mathrm{m}} 48 \mathrm{~s} .84, \delta(2000)$ $=33^{\circ} 32^{\prime} 48^{\prime \prime} .9$, just $0^{\prime \prime} 4$ from the measured position of the VLBA radio source (Wrobel et al. 2001) that is coincident with the optical nucleus. The X-ray source offset is well within the $\sim 1$." 5 Chandra position uncertainty, which should lay to rest any lingering doubt about the association between the central X-ray emission and the nucleus of NGC 4395. The radial profile of the nuclear X-ray source indicates that it is unresolved; the fractional encircled energy within a $2^{\prime \prime}$ radius is about $97 \%$.

A $5^{\prime \prime}$ radius aperture was used to extract light curves and the spectrum of the nucleus. The background was measured in a concentric annulus with inner and outer radii of $20^{\prime \prime}$ and $40^{\prime \prime}$, respectively. The background annulus lies between the nucleus and the nearest off-nuclear source (source B in Fig. 1). A net total of 2374 counts were detected in the $0.3-10.0 \mathrm{keV}$ energy range. Note that this is a factor of 20 greater than the number of counts detected in the ROSAT PSPC exposure of NGC 4395 (Moran et al. 1999), which had almost exactly the same duration.

\section{X-ray Variability}

Previous X-ray observations of NGC 4395 have established the variable nature of its nucleus. In the ROSAT band, the nuclear source varied by a factor of three over the several days spanning

\footnotetext{
${ }^{7}$ As a result of the subarray employed in our $17 \mathrm{ks}$ exposure, a bright nearby source (source E) was located outside the field of view. It was, however, included within the field of a short $1260 \mathrm{~s}$ ACIS-S observation of NGC 4395 (see Ho et al. 2001), which we have collected from the Chandra data archive. Figure 1 is a superposition of the two datasets; the true strength of source $\mathrm{E}$ is therefore not represented in the figure.
} 
the PSPC observation (Moran et al. 1999). Rapid variability (with doubling times on the order of $100 \mathrm{~s}$ ) was witnessed during the ASCA observation at energies greater than $2 \mathrm{keV}$, where the nucleus was more clearly isolated from other sources in the field (Iwasawa et al. 2000). Our high signal-to-noise ratio $(S / N)$ Chandra data are free of contamination over the broad $0.3-10 \mathrm{keV}$ range, and unlike the ROSAT and ASCA observations, the Chandra exposure is continuous.

The full-band light curve for the nucleus of NGC 4395 is displayed in Figure 2. A bin size of $77 \mathrm{~s}$ (= 50 frame times) has been employed. As the figure indicates, the source was highly variable during the $17 \mathrm{ks}$ Chandra observation; the count rate fluctuated by an order of magnitude during the exposure, with dramatic changes (factors of $2-3$ ) occurring over very short periods. While some of the strong flares and dips in the light curve [e.g., the flickering in the $t \approx(0.7-1.3) \times 10^{4}$ s range] appear to be time-resolved at this binning, there are a few instances where the count rate increased and then decreased (or vice versa) by a factor of $\sim 2$ over the span of a single $77 \mathrm{~s}$ bin. This time scale constrains the size of the emitting region to be less than 77 light-seconds $\left(\sim 2 \times 10^{12} \mathrm{~cm}\right)$.

To investigate whether the variability of the source has any spectral dependence, we have constructed light curves in different energy bands: hard (2-10 keV; "H"), medium (1-2 keV; "M"), and soft (0.3-1.2 keV; "S"). As shown in the top two panels of Figure 3, the hard-band and fullband light curves are very similar, both in terms of the features present and the total intensity. Thus, most of the detected source counts have energies above $2 \mathrm{keV}$. However, the light curves in the medium and soft energy bands (third and fourth panels of Fig. 3) provide important additional information about the variability properties of NGC 4395. While the count rates in all three bands are elevated in the $t \approx 7000-9000 \mathrm{~s}$ range, there are also clear differences between the $\mathrm{H}, \mathrm{M}$, and $\mathrm{S}$ light curves. Of particular interest are the dip in the $2-10 \mathrm{keV}$ count rate at $t \approx 5500 \mathrm{~s}$, which is absent in the soft and medium energy bands, and the strong increase in the soft-band count rate at $t \approx 11,500 \mathrm{~s}$, which is not apparent above $2 \mathrm{keV}$. These differences result in significant spikes in the X-ray "colors" of the source (i.e., the $\mathrm{S} / \mathrm{H}$ and $\mathrm{M} / \mathrm{H}$ count-rate ratios), which are shown in the bottom two panels of Figure 3. Sharp color variations are evident at other times as well, but in general they do not appear to correspond directly with changes in the $2-10 \mathrm{keV}$ count rate. As discussed in the following sections, these light curve properties play an important role in the interpretation of spectral models for the nuclear X-ray source.

\section{X-ray Spectroscopy}

X-ray spectroscopy provides vital clues about the emission and absorption components present in the nucleus of NGC 4395, as well as its intrinsic luminosity. Interesting spectral properties of this source have already been revealed in previous X-ray observations. For example, even though the $S / N$ of the ROSAT PSPC spectrum was poor, we were unable to obtain a good fit using a power-law model with absorption by a Galactic column of neutral material (Moran et al. 1999), which in the ROSAT band describes the X-ray spectra of most broad-line AGNs (e.g., Walter \& Fink 1993). The absence of strong spectral variability corresponding to the factor-of-three change 
in the source flux suggested to us that a single emission component dominates below $2 \mathrm{keV}$; we further speculated that an ionized (or "warm") absorber (Halpern 1984) might be responsible for the spectral complexity. The subsequent ASCA observation of NGC 4395 appeared to indicate an excess of soft X-ray emission during periods when the $2-10 \mathrm{keV}$ count rate was high. To account for this behavior, Iwasawa et al. (2000) adopted a model consisting of a power-law continuum and two components of ionized absorption, one that is constant and one that is variable. In this model, the ionization parameter of the latter component increases when the source is in an active state, resulting in a lower opacity in the $1-2 \mathrm{keV}$ range and excess observed flux in this band. Given the possibility that the ASCA spectrum was contaminated below $2 \mathrm{keV}$ by emission from nearby sources in the field, this scenario should be reexamined with the aid of Chandra's resolution.

The ACIS count rate is high enough (at times) to raise concerns about photon pile-up (the occurrence of two or more events in a pixel within one frame time), which could affect the shape of the observed spectrum. The first indication that pile-up is probably not significant in our observation of NGC 4395 is the fact that the strong edge in the mirror response near $2.1 \mathrm{keV}$ is present in the spectrum; this feature is usually smeared out when pile-up is severe. Simulations with the LYNX tool (Chartas et al. 2000; Eracleous et al. 2001) confirm that pile-up effects are minimal: the pile-up fraction is just $3 \%$ during periods of relative quiescence [e.g., the $t=(1.2-1.7) \times 10^{4} \mathrm{~s}$ range], and during most active phase (i.e., the $t=7000-9000 \mathrm{~s}$ range), the pile-up fraction is still only $\sim 10 \%$.

The bottom panels of Figure 3 indicate that the spectrum of NGC 4395 fluctuates somewhat with time. Although it would appear that spectral variations and features in the hard X-ray light curve are uncorrelated, the issue needs to be investigated further, since any dependence of the spectral properties on count rate would require us to model the high-state and low-state spectra separately. Unfortunately, given the degree of structure in the light curve shown in Figure 2, it is difficult to divide the data into temporally distinct active and quiescent phases, as Iwasawa et al. (2000) did for the ASCA observation. However, the source was clearly more active at certain times during the Chandra exposure than at others; we therefore extracted an "active" spectrum using data in the $t=6900-10,400 \mathrm{~s}$ range and a "quiescent" spectrum using data in the $t=1200-2400$, $5000-6000$, and $12,000-16,800$ s ranges. These spectra contain $28 \%$ and $20 \%$ of the total counts, respectively. As an alternative approach, we have derived the distribution of count rates in the light curve, which is displayed in Figure 4. Based on this histogram, we have compiled high-state and low-state spectra of the source by collecting counts in periods represented by the highest 6 bins $\left(>0.24\right.$ count s$\left.^{-1}\right)$ and the lowest 5 bins $\left(<0.15\right.$ count s$\left.{ }^{-1}\right)$ separately. These spectra contain $\sim 25 \%$ and $40 \%$ of the detected counts, respectively. In Figure 5 we have plotted the ratio of the high-state and low-state spectra derived using both of the methods described here. Despite the flux difference of a factor of $\sim 3$ between the two spectra in each case, spectral variations are only marginally significant. This is contrary to the results of the ASCA observation reported by Iwasawa et al. (2000). We note that the shape of the spectral ratio shown in Figure 5b, derived using the count-rate histogram in Figure 4, does bear some resemblance to a similar plot presented 
by Iwasawa et al. (2000). However, the 1-2 keV excess we observe relative to the $4-10 \mathrm{keV}$ ratio $(40 \%)$ is far less than the factor of $2-3$ excess indicated in the ASCA data. For the purposes of modeling the ACIS-S spectrum, we have no evidence that the spectrum changes significantly in direct response to count-rate fluctuations. Thus, in the analyses that follow, we model the spectrum derived from the full Chandra data set.

For model fitting, the Chandra spectrum of NGC 4395 was grouped into bins containing a minimum of 25 counts. Because of uncertainties in the ACIS-S response at the lowest and highest energies, we have ignored channels below $0.5 \mathrm{keV}$ and above $9 \mathrm{keV}$. A power-law model with absorption by neutral material provides a poor fit to the Chandra spectrum over the entire 0.5-9 $\mathrm{keV}$ range. It does, however, provide an excellent fit to the data above $\sim 1.2 \mathrm{keV}$. The best-fit photon index and absorption column density (with $90 \%$ confidence ranges for two parameters of interest) are $\Gamma=0.61_{-0.20}^{+0.25}$ and $N_{\mathrm{H}}=1.2_{-0.3}^{+0.4} \times 10^{22} \mathrm{~cm}^{-2}$; the fit is displayed in Figure 6 . An unresolved $\mathrm{Fe} \mathrm{K} \alpha$ emission line at $6.4 \mathrm{keV}$ is very marginally detected with an equivalent width of $99 \pm 95 \mathrm{eV}$ (90\% confidence). The improvement to the fit obtained with the inclusion of this component $\left(\Delta \chi^{2}=2.98\right.$ for one additional free parameter $)$ is significant at the $95 \%$ confidence level. Note that the power-law index we obtain is substantially lower than the value of $\Gamma=1.72$ obtained by Iwasawa et al. (2000) from their analysis of the ASCA observation. We return to this point in $\S 6.4$.

One means of explaining the apparent excess flux below $\sim 1 \mathrm{keV}$ would be to invoke a second emission component that dominates at low energies. We obtain an excellent fit over the full energy range if this additional component is an optically thin thermal plasma (either a Raymond-Smith or "MEKAL" component) with a temperature of $\sim 0.1 \mathrm{keV}$. However, as Figure 3 indicates, the source is quite variable in the $0.3-1.2 \mathrm{keV}$ band. The time scale of the variability, the temperature of the thermal component, and its implied luminosity combine to suggest that such a plasma would be optically thick (Elvis et al. 1991), which rules out composite models of this sort. We also tried composite models where the second component is either a blackbody or another (steeper) power law; such components could plausibly be associated with a compact emission region near the black hole. These models also provide good fits (although the residuals are more pronounced below 1 $\mathrm{keV}$ ). They do not, however, easily explain why the soft-band and hard-band light curves are similar in some respects and dissimilar in others. The most likely scenario, in our minds, is that a single emission component dominates over the entire $0.3-10 \mathrm{keV}$ band.

To investigate this possibility, we have refitted the Chandra spectrum above $2.2 \mathrm{keV}$ with a single power-law model, assuming only the Galactic neutral hydrogen column density $\left(1.4 \times 10^{20}\right.$ $\mathrm{cm}^{-2}$; Murphy et al. 1996). The residuals associated with this fit, displayed in Figure 7, exhibit several strong dips in the soft X-ray band. The deepest feature at $\sim 0.8 \mathrm{keV}$ is consistent with the absorption edges of O VII $(0.74 \mathrm{keV})$ and O VIII $(0.85 \mathrm{keV})$, which suggests that an ionized absorber (Halpern 1984; Reynolds 1997) is present in NGC 4395. Indeed, with the addition of a warm absorber to the model (i.e., the "absori" component in the XSPEC spectral analysis package), excellent fits to the Chandra spectrum over the full energy range are possible. Assuming a fixed 
temperature of $1 \times 10^{5} \mathrm{~K}$ for the absorber, we obtain an acceptable fit $\left(\chi^{2}=67.6\right.$ for 79 degrees of freedom) with the following model parameters: a power-law photon index $\Gamma=0.54$, a column density of the ionized medium $N_{\text {warm }}=1.37 \times 10^{22} \mathrm{~cm}^{-2}$, and an ionization parameter $\xi=6.4 \mathrm{erg}$ $\mathrm{cm} \mathrm{s}^{-1}$. A slightly better fit $\left(\chi^{2}=63.7\right)$ is obtained assuming $T=5 \times 10^{4} \mathrm{~K}$; the fit parameters (with uncertainties corresponding to $\Delta \chi^{2}=2.71$ ) are $\Gamma=0.56_{-0.13}^{+0.13}, N_{\text {warm }}=1.49_{-0.29}^{+0.31} \times 10^{22}$ $\mathrm{cm}^{-2}$, and $\xi=9.3_{-3.1}^{+4.1} \mathrm{erg} \mathrm{cm} \mathrm{s}^{-1}$. This best-fit model is displayed in Figure 8. In general, as the assumed temperature is increased, the model ionization parameter decreases and the fit below 1.3 $\mathrm{keV}$ worsens.

Although not required by the data, we have also fitted a model consisting of two components of ionized absorption similar to the multizone warm absorber model used by Iwasawa et al. (2000). The temperatures of the two components are assumed to be $5 \times 10^{4} \mathrm{~K}$ and $1 \times 10^{6} \mathrm{~K}$. Fixing the photon index at a value of 0.56 , the improvement to the fit obtained with this model $\left(\Delta \chi^{2}=2.54\right.$ for one additional free parameter) is significant at the $92 \%$ confidence level. The column density and ionization parameter of the "cool" warm absorber component are $N_{\text {warm }}=1.0 \times 10^{22} \mathrm{~cm}^{-2}$ and $\xi=4.6 \mathrm{erg} \mathrm{cm} \mathrm{s}^{-1}$. For the "hot" component, we find $N_{\text {warm }}=1.4 \times 10^{22} \mathrm{~cm}^{-2}$ and $\xi=42 \mathrm{erg} \mathrm{cm}$

$\mathrm{s}^{-1}$. Given the flexibility of the model and the quality of the data, it is not too surprising that these parameters are not very well constrained. Additional observations with higher $S / N$ are needed to give us a better handle on the physical state of the ionized gas in NGC 4395, particularly if the absorption varies with time, as the difference between our results and those obtained with $A S C A$ might suggest. The simple fact that warm absorber models afford such good fits is important, since it supports the notion that the broadband X-ray spectrum of NGC 4395 is dominated by a single emission component. The extremely flat slope of this component, however, is very surprising; in the next section we consider a variety of possibilities for its origin.

\section{Implications for the Nature of the Nucleus of NGC 4395}

\subsection{Broadband Luminosity and Variability of the Source}

Using the high angular resolution of Chandra, we have established that the central X-ray source in NGC 4395 is coincident with the galaxy's optical and radio nucleus. The X-ray emission is spatially unresolved and highly variable, leaving no doubt that it is associated with the dwarf AGN in the galaxy. Provided that a single emission component dominates in the Chandra band, the power-law fit to the ACIS-S spectrum above $1.2 \mathrm{keV}$ suggests an absorption-corrected, timeaveraged 2-10 keV luminosity of $8.0 \times 10^{39} \mathrm{ergs} \mathrm{s}^{-1}$ for an adopted distance of $4.1 \mathrm{Mpc}$. This is within $25 \%$ of the value obtained with $A S C A$ (adjusted for this distance) by Iwasawa et al. (2000). In the $0.5-10 \mathrm{keV}$ band, $L_{\mathrm{X}}=9.0 \times 10^{39} \mathrm{ergs} \mathrm{s}^{-1}$. Combining the broadband X-ray luminosity with the radio-to-UV luminosity computed by Moran et al. (1999, after revision for the 4.1 Mpc distance), we obtain a bolometric luminosity of $L_{\mathrm{bol}}=5.3 \times 10^{40} \mathrm{ergs} \mathrm{s}^{-1}$. This luminosity corresponds to an Eddington-limit mass of $\sim 400 M_{\odot}$ for the accreting black hole. As reported by 
Filippenko \& Ho (2002), the conservative upper limit for the black-hole mass is $8 \times 10^{5} M_{\odot}$; the actual mass, they argue, is likely to be between $\sim 1 \times 10^{4} M_{\odot}$ and $\sim 1 \times 10^{5} M_{\odot}$. Using this range of values, the bolometric luminosity suggests an Eddington ratio of $L_{\mathrm{bol}} / L_{\text {edd }}=0.004-0.04$.

The nucleus of NGC 4395 is an exceptionally variable X-ray source. As pointed out by Iwasawa et al. (2000), such extreme variability is quite uncharacteristic of low-luminosity AGNs (LLAGNs). The "excess variance" (Nandra et al. 1997; Turner et al. 1999) of the source in the $2-10 \mathrm{keV}$ band is $0.35 \pm 0.03$ (using $128 \mathrm{~s}$ bins), almost twice that estimated by Iwasawa et al. using ASCA data and far greater than the excess variances measured for other LLAGNs (e.g., Ptak et al. 1998). However, many of the LLAGNs examined to date are LINERs, which are likely to be accreting in an advection-dominated mode (e.g., Lasota et al. 1996; Ho 1999) — in other words, they are supermassive black holes $\left(M>10^{6} M_{\odot}\right)$ with low accretion rates $\left(\dot{M} / \dot{M}_{\text {edd }}<10^{-3}\right)$. In contrast, NGC 4395 appears to possess a smaller black hole that is accreting at a higher rate.

\subsection{Ionized Absorption and the Short-Term Spectral Variability}

The X-ray spectrum of NGC 4395 below $\sim 1.2 \mathrm{keV}$ is complex, indicating that either a second emission component dominates at low energies or that an ionized absorber is present. The rapid variability of the source in the soft X-ray band, and the fact that some of the count-rate fluctuations observed above $2 \mathrm{keV}$ occur at lower energies as well, argue for the latter interpretation. We obtain an excellent fit to the ACIS-S spectrum with a model consisting of a power-law continuum, neutral absorption equivalent to the Galactic column, and a single-temperature component of ionized absorption. If a simple, constant warm absorber were present, however, we would expect the light curve below $1 \mathrm{keV}$ to closely resemble the hard-band light curve, which it does not. On the other hand, the more complex multizone warm absorber model proposed by Iwasawa et al. (2000) cannot explain our results either. In that scenario, the opacity of a variable absorption component changes in response to intensity fluctuations of the source, which leads to excess soft X-ray emission when the source is in a high-flux state. The Chandra light curves in Figure 3 and the spectral ratio plots shown in Figure 5 do not indicate this sort of behavior. In general, excess soft X-ray emission does not accompany an increase in the hard-band count rate, and in at least one instance, we observe a dramatic softening of the spectrum corresponding to a sharp decrease in the hard X-ray flux.

A possible explanation for the short-term spectral variability we observe is that the absorbing medium in NGC 4395, rather than being physically variable, is geometrically variable or clumpy. The spatial scale of the clumpiness and the transverse velocity of the medium could, in the right combination, give rise to rapid column density variations along the line of sight, and thus, to changes in the observed soft X-ray flux that are partially independent of the continuum variability above $2 \mathrm{keV}$. Soft-band and hard-band light curves that are similar in some respects, but not in others, would be a natural consequence of this scenario. As a test, we have used the PIMMS software to compute the ACIS-S count rates that would be observed in various bands if the $\Gamma=0.6$ power law were absorbed only by a Galactic column of neutral material. Relative to the 2-10 
$\mathrm{keV}$ band, we would expect 0.65 and 0.73 times as many counts in the $0.3-1.2 \mathrm{keV}$ and $1-2 \mathrm{keV}$ bands, respectively. If a clumpy absorbing medium in NGC 4395 is responsible for differences in the soft-band and hard-band light curves, the X-ray colors of the source defined in Figure 3 should not exceed these amounts. Referring to the figure, we see that the maximum value of Color 1 (the [0.3$1.2 \mathrm{keV}] /[2-10 \mathrm{keV}]$ ratio) is $\sim 0.4$ and the maximum value of Color 2 (the $[1-2 \mathrm{keV}] /[2-10 \mathrm{keV}]$ ratio) is $\sim 0.7$, consistent with our hypothesis. To further investigate the role of column density variations, we have used the Color 1 light curve to predict the values of Color 2 for the entire Chandra observation. This was done by adjusting the column density of the ionized medium in the best-fit single warm absorber model (that shown in Fig. 8) until the observed values of Color 1 were obtained. The same model, with the modified column density, was then used to derive Color 2 . The range of column densities implied is $\sim 1 \times 10^{22}$ to $\sim 4 \times 10^{22} \mathrm{~cm}^{-2}$. As Figure 9 illustrates, the agreement between the observed and predicted values of Color 2 is quite good. Unfortunately, the uncertainties are rather substantial, and it is too soon to conclude that column density variations alone are responsible for the X-ray color changes observed in NGC 4395. These results, however, are encouraging.

\subsection{Origin of the Hard X-ray Continuum}

Although a power law provides a perfectly adequate description of the hard-band spectrum, the spectral index we measure $(\Gamma \approx 0.6)$ is substantially flatter than the typical slopes of AGN spectra, which are generally in the $\Gamma \approx 1.7-1.9$ range (e.g., Nandra \& Pounds 1994). To our knowledge, an intrinsic X-ray spectrum as flat as that of NGC 4395 has never been observed in an AGN. Thus, it would be very worthwhile to investigate the reason the spectrum of this source is so hard. First, we consider the possibility that the nuclear spectrum of NGC 4395 is intrinsically flat. It is generally believed that hard X-rays in AGNs are produced by low-energy photons (perhaps from an accretion disk) that are inverse-Compton scattered by a corona of hot $(\sim 100 \mathrm{keV})$ thermal electrons with fairly low optical depth. This mechanism, however, cannot yield a spectrum with a photon index less than 1; a flatter spectrum would imply that scattered photons at higher energies have acquired more energy per scattering than those at lower energies, which is impossible. If the scattering optical depth is large, a flat spectrum in the band corresponding to the Wien peak of the electron energy distribution is possible. This circumstance, however, implies that the photons undergo many scatterings, which would smear out the rapid variability we observe (unless the emitting region is much smaller than the limit set by the variability time scale see $\S 4$ ). Comptonization by a nonthermal population of electrons cannot be ruled out, although an extreme electron energy spectrum would be required in this scenario. For a power-law distribution of electron energies with index $p$, the energy index of inverse-Compton radiation $\alpha(=\Gamma-1)$ is $(p-1) / 2$ (e.g., Tucker 1975). Thus, for $\Gamma=0.6$, a $p=0.2$ electron spectrum is required - vastly different from the $p \approx 2.2$ spectrum inferred for the electrons that produce the compact synchrotron radio emission in the nucleus of NGC 4395 (Ho \& Ulvestad 2001). Thermal bremsstrahlung emission from a hot, optically

thin plasma produces a very flat spectrum at X-ray energies below the characteristic temperature 
$k T$ of the plasma. However, bremsstrahlung models (even those with very high temperatures) have too much curvature to provide good fits to the spectrum of NGC 4395 above $2 \mathrm{keV}$. Furthermore, the small size of the source set by the variability and the implied emissivity suggest a large optical depth in the plasma, which firmly rules out bremsstrahlung emission (e.g., Elvis et al. 1991).

Alternatively, perhaps the intrinsic spectrum of NGC 4395 is steep, but modified in such a way that a flat spectrum is observed. Compton reflection (e.g., Matt et al. 1996) is an obvious candidate for this effect; reprocessing by "cold" material can result in a very flat observed spectrum if the primary steep-spectrum emission from the source is obscured. Two factors lead us to conclude that reflection does not provide a viable solution. First, reflection-dominated spectra should be accompanied by a strong (1-2 keV equivalent width) Fe $\mathrm{K} \alpha$ emission line at $6.4 \mathrm{keV}$, but there is no evidence for such a strong line in the spectrum of NGC 4395 (Fig. 6). A second constraint is provided by the rapid variability of the source, which implies a maximum source size that is only somewhat larger than the region where the primary hard X-ray emission is expected to originate $\left(\sim 6 \times 10^{11} \mathrm{~cm}\right.$, if $M_{\mathrm{BH}}=10^{5} M_{\odot}$ and the hard X-rays are produced within about 10 Schwarzschild radii). For reflection to dominate, obscuration would have to cover the primary emission but not the reflector, which seems unlikely. Another possibility is that NGC 4395 has an intrinsically steep spectrum that is flattened by a complex neutral absorber consisting of multiple "partial covering" components. In this scenario, a fraction of the steep-spectrum emission is seen directly, but the majority is observed via transmission through different columns of dense material. The result is a flat observed spectrum that has a roughly power-law form (e.g., Zdziarski et al. 2001). Indeed, we obtain a very good fit to the spectrum of NGC 4395 above $1.2 \mathrm{keV}$ with a model consisting of a $\Gamma=1.7$ power law absorbed by three partial-covering components. The absorbers in this model have fairly high columns $\left(1-90 \times 10^{22} \mathrm{~cm}^{-2}\right)$ and covering fractions $(0.5-0.9)$. Interestingly, we find that this model provides a reasonable fit to the entire ACIS-S spectrum. The fit below $1.2 \mathrm{keV}$ is not as good as that obtained with a single warm absorber, but this is easily rectified with the addition of a modest amount of ionized absorption. The intrinsic $2-10 \mathrm{keV}$ luminosity of the source implied in this model is higher than the one we reported above for a $\Gamma=0.6$ power law, but only by a factor of 2.5. We note, however, that this model predicts a strong Fe $\mathrm{K}$ edge near $7 \mathrm{keV}$, which does not appear to be present in the Chandra spectrum.

\subsection{Dramatic Long-Term Spectral Variability}

Of great importance is the possibility that the shape of the broadband continuum in NGC 4395 has undergone a dramatic change during the two years between the ASCA and Chandra observations. This is implied by the difference in the power-law photon index we measure $(\Gamma \approx 0.6)$ and that obtained with $A S C A(\Gamma \approx 1.7)$ by Iwasawa et al. $(2000)$. Such behavior could provide important new insight into the accretion processes not only in NGC 4395, but perhaps generally in active galactic nuclei.

As a first step, we have examined the spectrum of the nuclear source in NGC 4395 during 
the short 1260 s Chandra exposure obtained by Ho et al. (2001), which was carried out about two months before our $17 \mathrm{ks}$ observation. Despite the limited $S / N$ ratio of the snapshot spectrum, an absorbed power-law model provides a good fit above $\sim 1.2 \mathrm{keV}$; we find that $\Gamma<1.0(90 \%$ confidence). Thus, we need to consider the possibility that the spectrum of the nuclear emission is always flat and appears steep in the ASCA data due to contamination by nearby sources in the field. The large $6^{\prime} \times 6^{\prime}$ SIS extraction area used by Iwasawa et al. to investigate the spectrum above $2 \mathrm{keV}$ is approximately the same region shown here in Figure 1. They omitted a small region centered on source $\mathrm{E}$, but estimated that $\sim 30 \%$ of the counts from $\mathrm{E}$ were included in the extraction. To approximate the ASCA spectrum, we have summed the emission from the nucleus and all of the other resolved sources in Figure 1. The contribution of source E, which was present in the $1260 \mathrm{~s}$ exposure but not our 17 ks observation, was determined by scaling the number of counts in the snapshot spectrum by the exposure-time ratio and then multiplying by 0.3 . We find that in the $0.3-10 \mathrm{keV}$ band, only $62 \%$ of the counts in the simulated ASCA spectrum are associated with the nucleus; $16 \%$ of the counts are associated with source E, and the remaining $22 \%$ are from the other resolved sources. However, most of the contamination occurs at low X-ray energies - a power-law fit to the simulated $A S C A$ spectrum above $2 \mathrm{keV}$ indicates a flat spectral index similar to that which we measured for the nucleus alone. We conclude, therefore, that if the spectrum of the nucleus was flat during the ASCA observation, it would have appeared as such to Iwasawa et al., implying that the slope of the broadband continuum of NGC 4395 is indeed capable of dramatic variations.

The other significant difference between our results and those obtained with ASCA concerns the variability of the nucleus in the $1-2 \mathrm{keV}$ band and its dependence on the source count rate at higher energies. A strong excess $1-2 \mathrm{keV}$ flux associated with increases in the $2-10 \mathrm{keV}$ count rate was seen in the ASCA data (Iwasawa et al. 2000), but not in the Chandra observation presented here. Contamination of the soft-band ASCA spectrum is severe - we find that over $60 \%$ of the counts below $2 \mathrm{keV}$ in the large SIS aperture employed by Iwasawa et al. are associated with contaminating sources - providing a possible explanation for the different results. On the other hand, the 1-2 keV excess reported by Iwasawa et al. was measured in SIS spectra extracted with a smaller $3^{\prime}$ diameter aperture, which would have had a reduced level of contamination. In view of the drastic difference we observe in the slope of the nuclear spectrum and the possibility of column density variations in NGC 4395 (see above), different behavior in the 1-2 keV band during the ASCA observation seems entirely plausible. This and other issues regarding the nature of NGC 4395 should be explored with future high-resolution X-ray observations.

\section{Summary}

The nucleus of NGC 4395 is unique among type 1 AGNs. In addition to having one of the lowest observed luminosities and least massive black holes, it displays a number of extreme properties in the X-ray band as well. As our Chandra observation confirms, NGC 4395 has a spectacular X-ray 
light curve that exhibits large-amplitude variations on extremely short time scales. This object is one of the most X-ray-variable AGNs known. It appears that ionized gas is responsible for the bulk of the X-ray absorption in NGC 4395; variations in the column density of this material may be the cause of the short-term spectral variability we detect over the course of the Chandra exposure.

But the most extreme aspect of the nuclear X-ray source in NGC 4395 is the slope of its hard $\mathrm{X}$-ray spectrum, which is modeled as a $\Gamma=0.6$ power law. This is significantly flatter than the X-ray spectral slopes associated with more luminous type 1 AGNs $(\langle\Gamma\rangle \approx 1.8)$. Unfortunately, it remains unclear why the spectrum of NGC 4395 is so flat - Comptonization requires an extremely small, optically thick scattering region or an electron population with very unusual energetics, and none of the alternative possibilities we have explored (e.g., multiple partial-covering absorption components) provides a fully satisfactory explanation for the flat slope. Even more intriguing, however, are the dramatic changes in the X-ray absorption and spectral characteristics of NGC 4395 that have apparently occurred during the two years between the ASCA and Chandra observations. Analysis of the ASCA data by Iwasawa et al. (2000) revealed a "normal" X-ray spectral slope of $\Gamma=1.7$ for NGC 4395 and excess flux in the $1-2 \mathrm{keV}$ band corresponding to increases in the 2-10 keV count rate, neither of which is evident in the Chandra data. Our preliminary analysis suggests that contamination of the nuclear emission in the ASCA image by nearby sources is not responsible for these differences. If so, we must conclude that the nucleus of NGC 4395 undergoes dramatic changes in the slope of its continuum and/or its absorption properties on relatively short time scales. Spectral variations of this magnitude have never been observed in an AGN.

Obviously, X-ray monitoring of NGC 4395 with high-resolution instrumentation is crucial for a clarification of the physical processes occurring in its nuclear region. Ultimately, a firm understanding of the unusual behavior in this object may provide general insight into the physics of AGNs.

We are grateful to Andrzej Zdziarski for helpful discussions about X-ray continuum models. E.C.M. is supported by NASA through Chandra Fellowship grant PF8-10004 awarded by the Chandra X-ray Center, which is operated by the Smithsonian Astrophysical Observatory for NASA under contract NAS 8-39073. A.V.F. thanks NASA for financial support through Chandra grant GO0-1170A, and the Guggenheim Foundation for a fellowship.

\section{REFERENCES}

Chartas, G., et al. 2000, ApJ, 542, 655

Elvis, M., Wilkes, B. J., Giommi, P., \& McDowell, J. 1991, ApJ, 378, 537

Eracleous, M., Shields, J. C., Chartas, G., \& Moran, E. C. 2001, ApJ, in press

Filippenko, A. V., \& Ho, L. C. 2002, ApJ, submitted 
Filippenko, A. V., Ho, L. C., \& Sargent, W. L. W. 1993, ApJ, 410, L75

Filippenko, A. V., \& Sargent, W. L. W. 1989, ApJ, 342, L11

Halpern, J. P. 1984, ApJ, 281, 90

Ho, L. C. 1999, ApJ, 516, 752

Ho, L. C., et al. 2001, ApJ, 549, L51

Ho, L. C., \& Ulvestad, J. S. 2001, ApJS, 133, 77

Iwasawa, K., Fabian, A. C., Almaini, O., Lira, P., Lawrence, A., \& Hyashida, K. 2000, MNRAS, 318,879

Kraemer, S. B., Ho, L. C., Crenshaw, D. M., Shields, J. C., \& Filippenko, A. V. 1999, ApJ, 520, 564

Lasota, J.-P., Abramowicz, M. A., Chen, X., Krolik, J., Narayan, R., \& Yi, I. 1996, ApJ, 462, 142

Lira, P., Lawrence, A., O’Brien, P., Johnson, R. A., Terlevich, R., \& Bannister, N. 1999, MNRAS, 305, 109

Matt, G., et al. 1996, MNRAS, 281, L69

Minitti, D., et al. 2002, in preparation

Moran, E. C., Filippenko, A. V., Ho, L. C., Shields, J. C., Belloni, T., Comastri, A., Snowden, S. L., \& Sramek, R. A. 1999, PASP, 111, 801

Murphy, E. M., Lockman, F. J., Laor, A., \& Elvis, M. 1996, ApJS, 105, 365

Nandra, K., George, I. M., Mushotzky, R. F., Turner, T. J., \& Yaqoob, T. 1997, ApJ, 476, 70

Nandra, K., \& Pounds, K. A. 1994, MNRAS, 268, 405

Ptak, A., Yaqoob, T., Mushotzky, R., Serlemitsos, P., \& Griffiths, R. 1998, ApJ, 501, L37

Reynolds, C. S. 1997, MNRAS, 286, 513

Tucker, W. H. 1975, Radiation Processes in Astrophysics (Cambridge: MIT Press), 168

Turner, T. J., George, I. M., Nandra, K., \& Turcan, D. 1999, ApJ, 524, 667

Walter, R,. \& Fink, H. H. 1993, A\&A, 274, 105

Wrobel, J. M., Fassnacht, C. D., \& Ho, L. C. 2001, ApJ, 553, L23

Zdziarski, A. A., Leighly, K. M., Matsuoka, M., Cappi, M., \& Mihara, T. 2001, ApJ, in press 


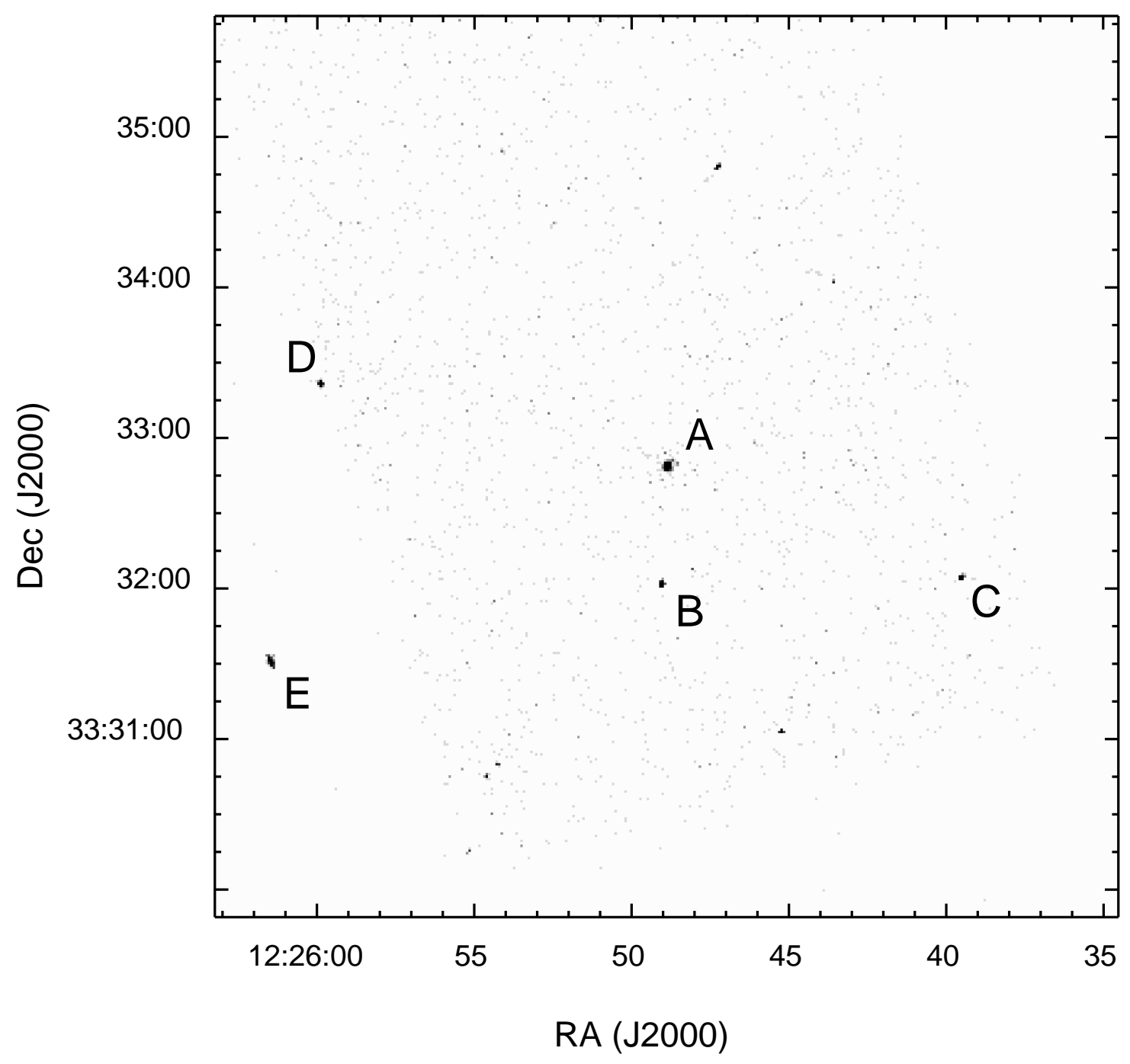

Fig. 1.- Chandra ACIS-S image of NGC 4395 in the $0.3-10 \mathrm{keV}$ band. Sources A-E were previously identified in a ROSAT PSPC observation (Moran et al. 1999); several other sources are visible as well. Source A is coincident with the nucleus of the galaxy. It is clear from this image that the nucleus is completely isolated from the other sources in the field, which was not the case in the ASCA observation of NGC 4395 (Iwasawa et al. 2000). 


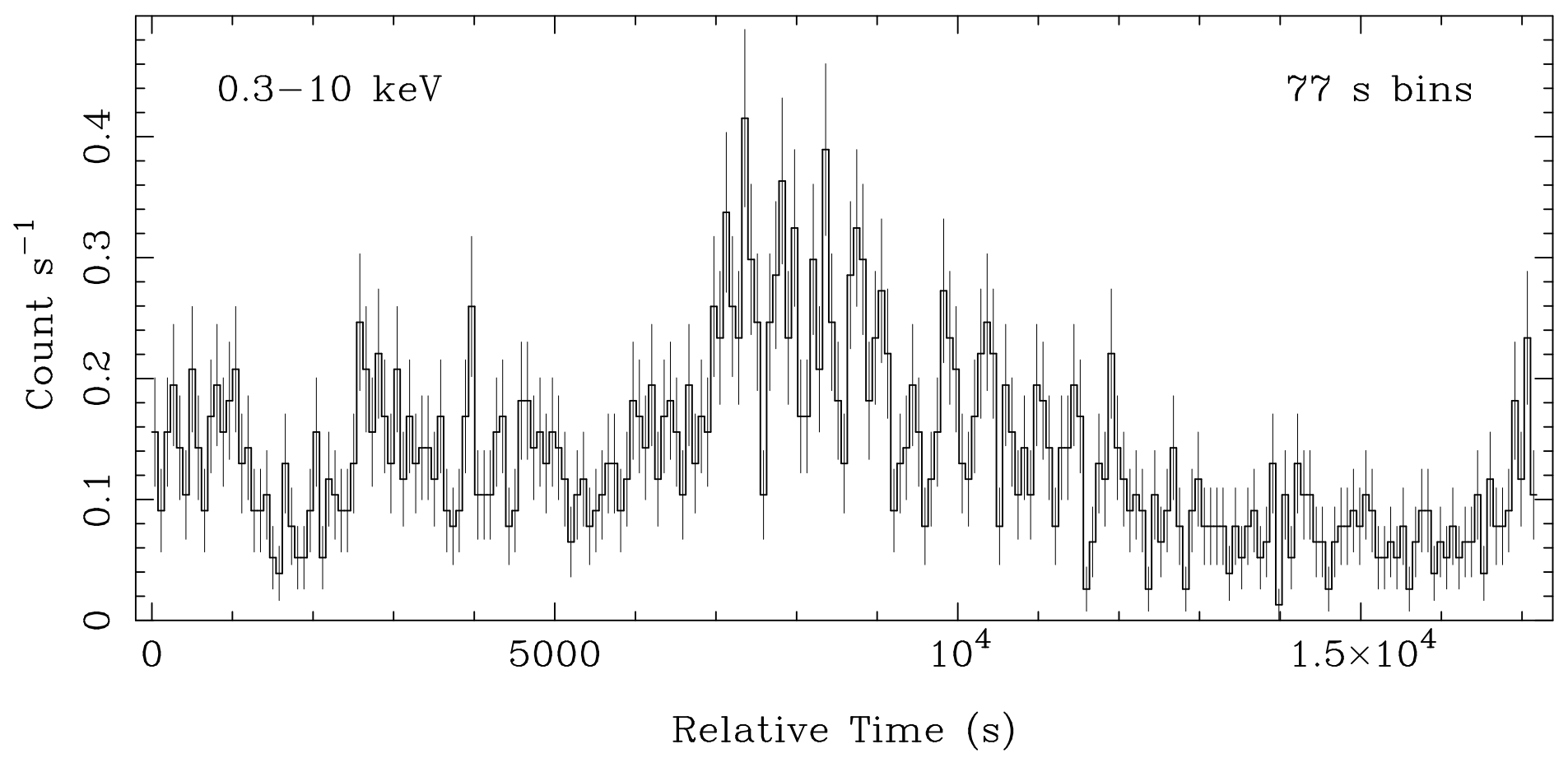

Fig. 2.- The 0.3-10 keV light curve of NGC 4395. Each $77 \mathrm{~s}$ bin corresponds to 50 frames. The source exhibits large-amplitude variability on very short time scales. 


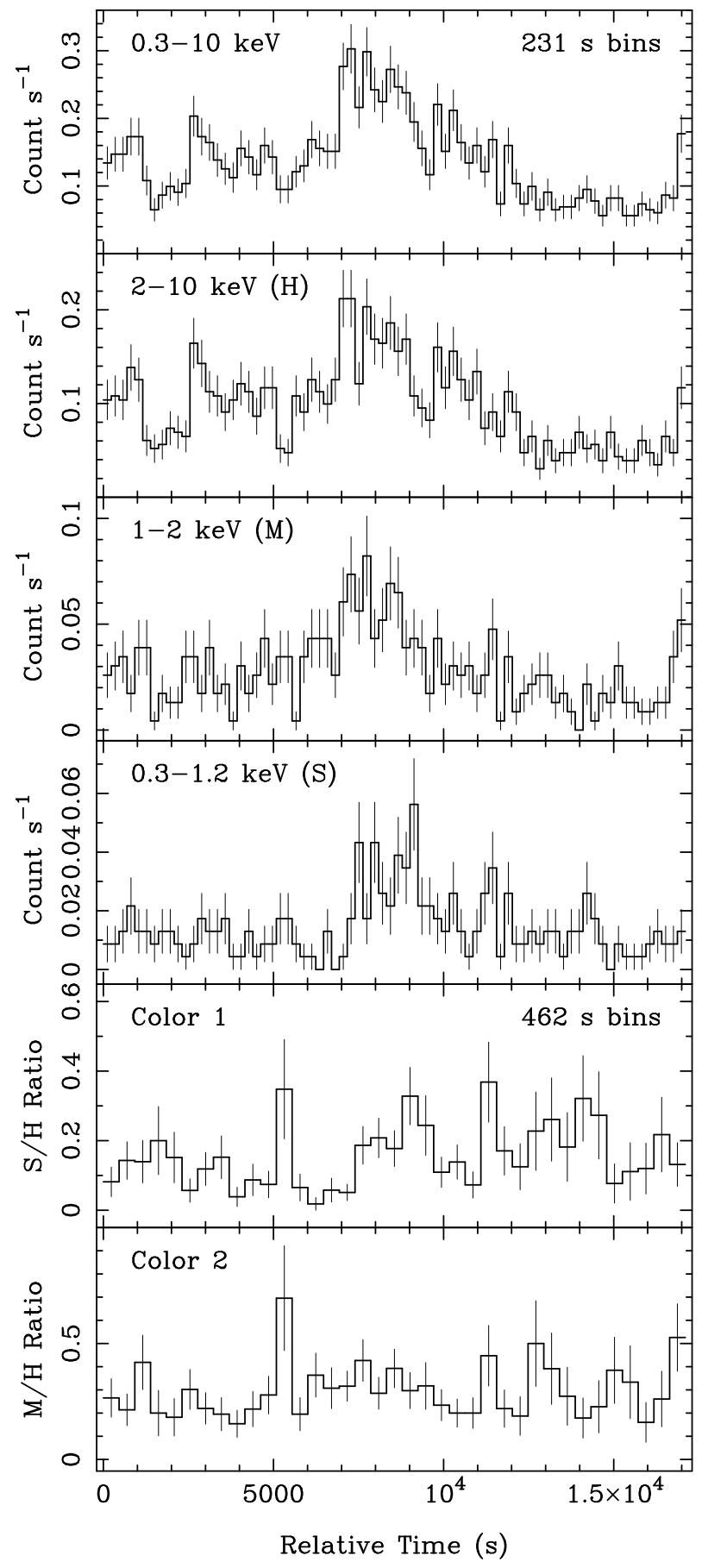

Fig. 3.- Light curves of NGC 4395 in broad, hard (H), medium (M), and soft (S) energy bands (top four panels). The $231 \mathrm{~s}$ bins correspond to 150 frames. The bottom two panels indicate the $\mathrm{S} / \mathrm{H}$ counts ratio (Color 1 ) and the $\mathrm{M} / \mathrm{H}$ counts ratio (Color 2) for the source in 300-frame bins. Note that there is little apparent correspondence between either color and the $2-10 \mathrm{keV}$ count rate. 


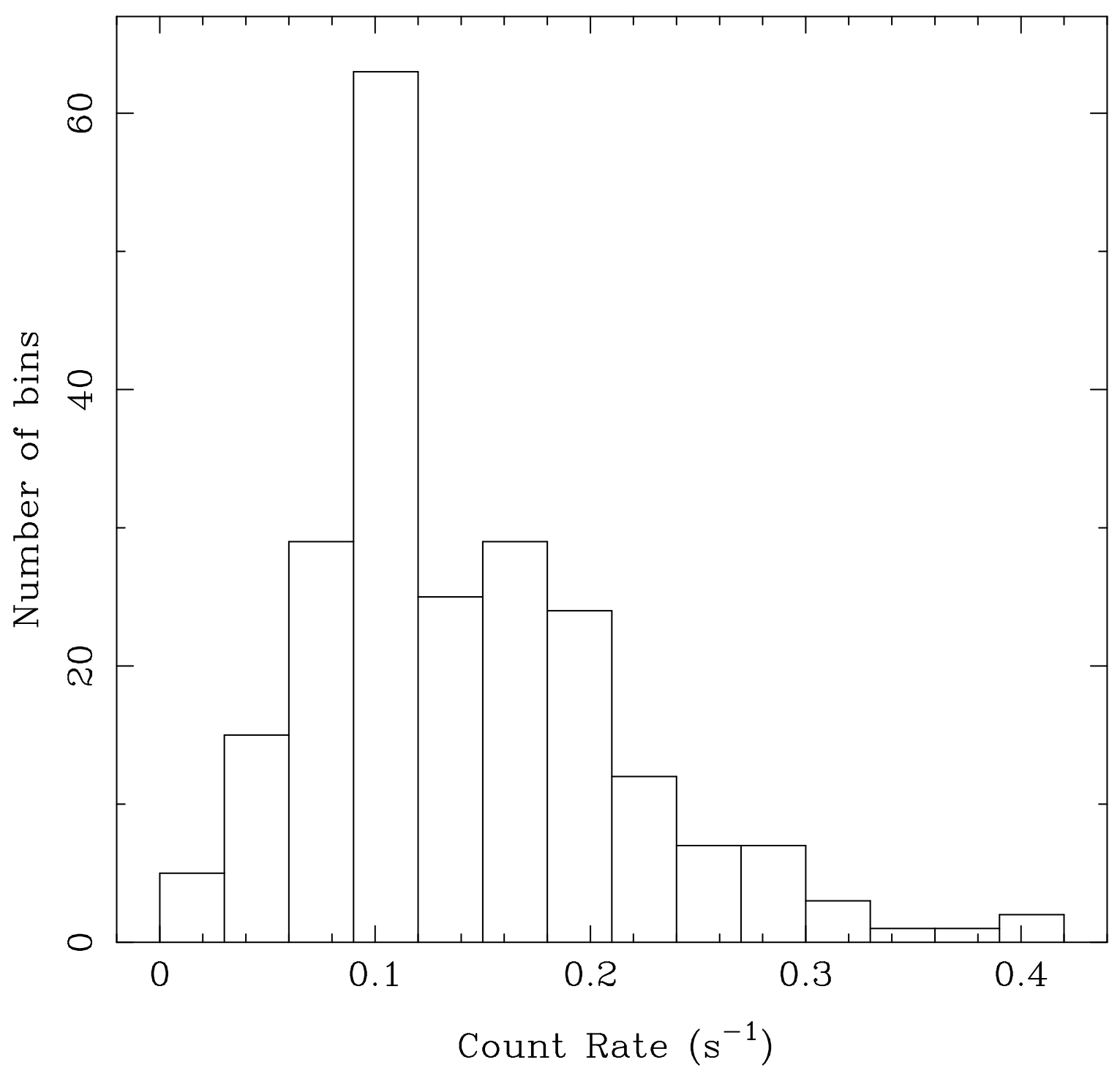

Fig. 4.- Distribution of count rates in the 50-frame light curve shown in Fig. 2. 


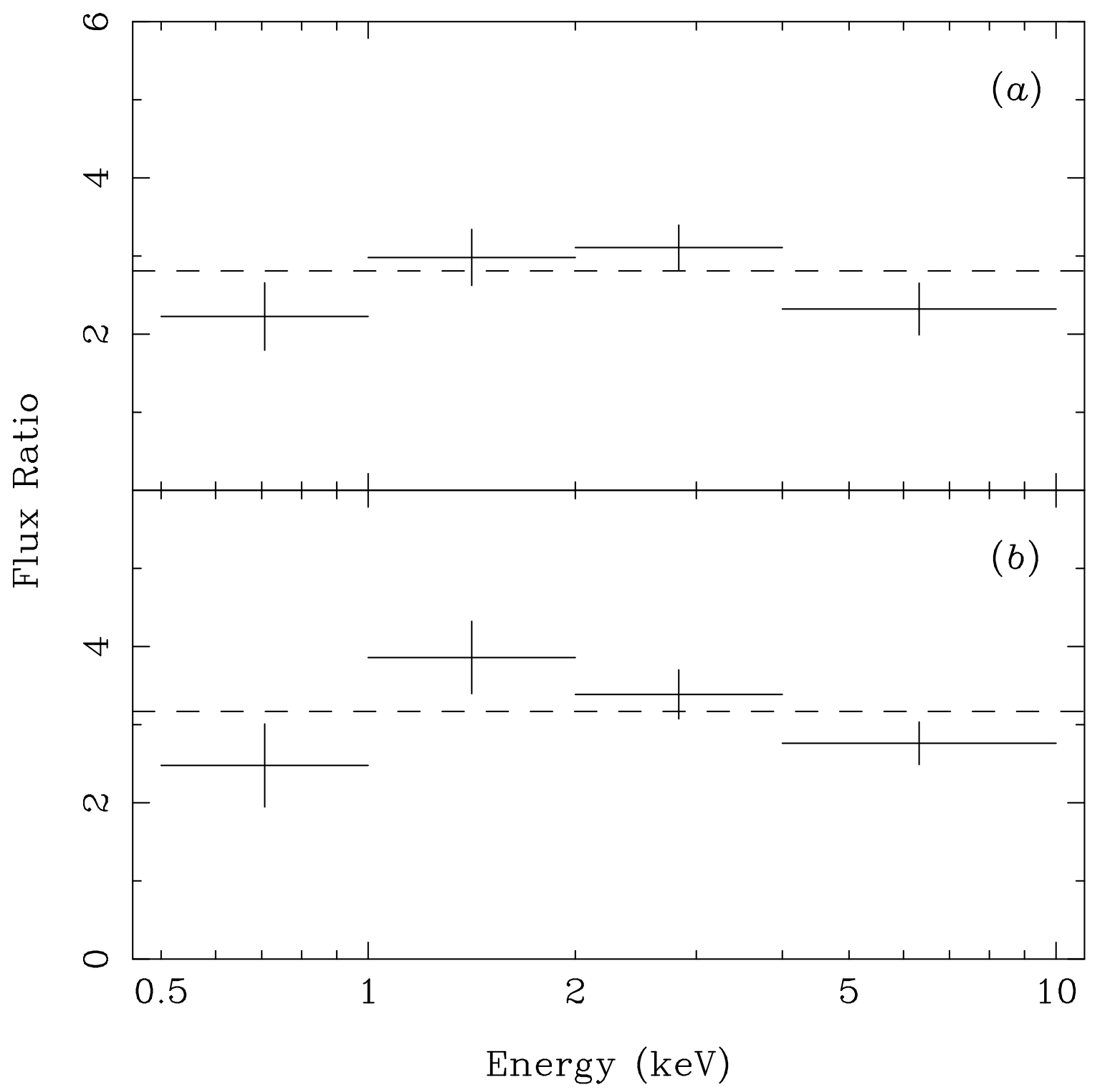

Fig. 5.- Ratio of spectra of NGC 4395 in "active" and "quiescent" states, which have been defined in two different ways (see the text for details). In $(a)$, the high-state and low-state spectra are associated with broad periods in the light curve during which the activity of the source was visibly different. For $(b)$, the high-state and low-state spectra were derived on the basis of the count-rate histogram shown in Fig. 4. The dashed lines denote the mean ratio in the two cases. Neither plot indicates strong spectral variability corresponding to the factor-of-three count rate changes. 


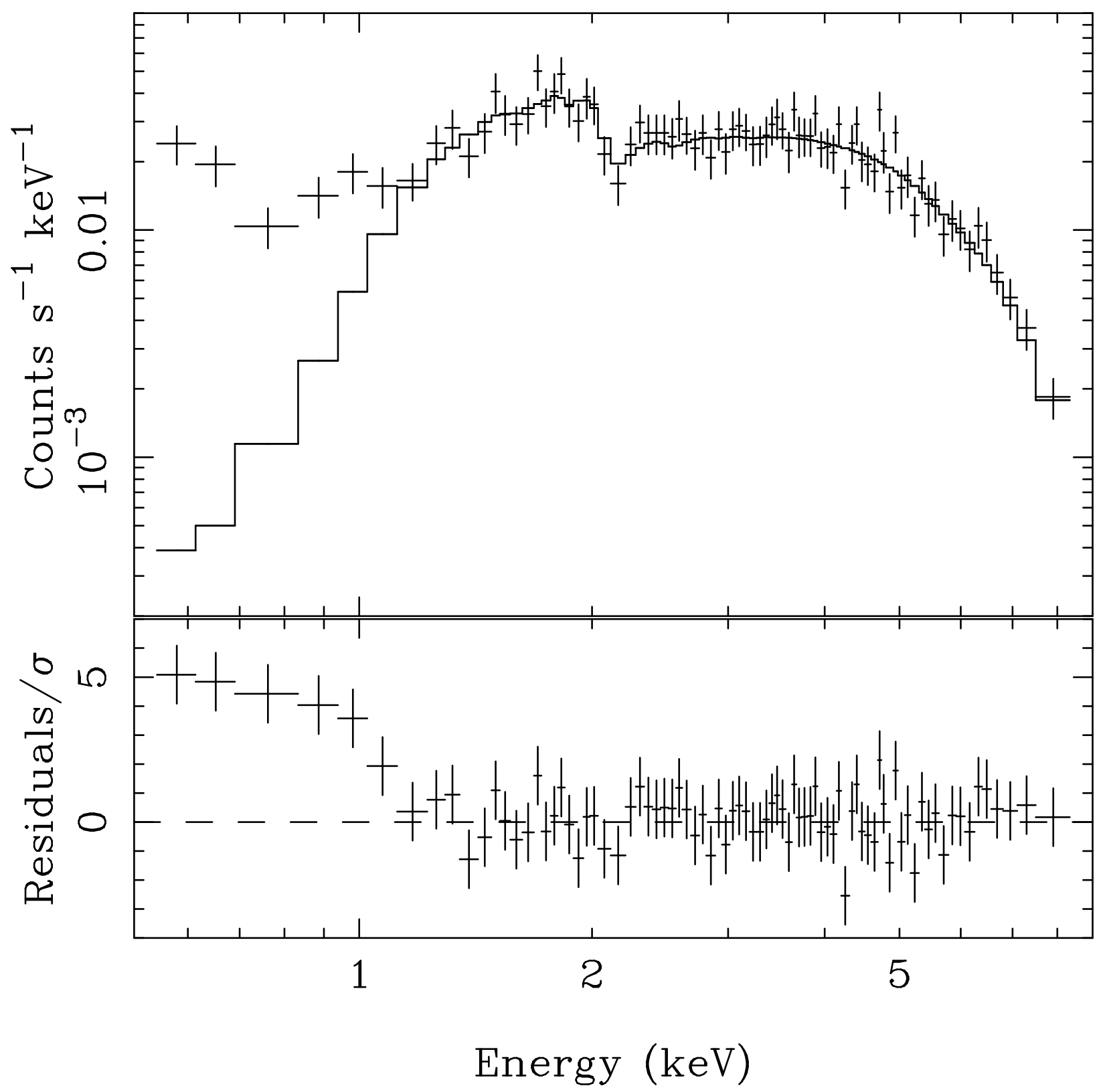

Fig. 6.- (top panel) Observed ACIS-S spectrum of NGC 4395, fitted with a simple absorbed power-law model above $1.2 \mathrm{keV}\left(\Gamma=0.61, N_{\mathrm{H}}=1.2 \times 10^{22} \mathrm{~cm}^{-2}\right)$. The fit residuals (normalized by the $1 \sigma$ errors) are shown in the lower panel. Note the strong excess flux at the lowest energies. 


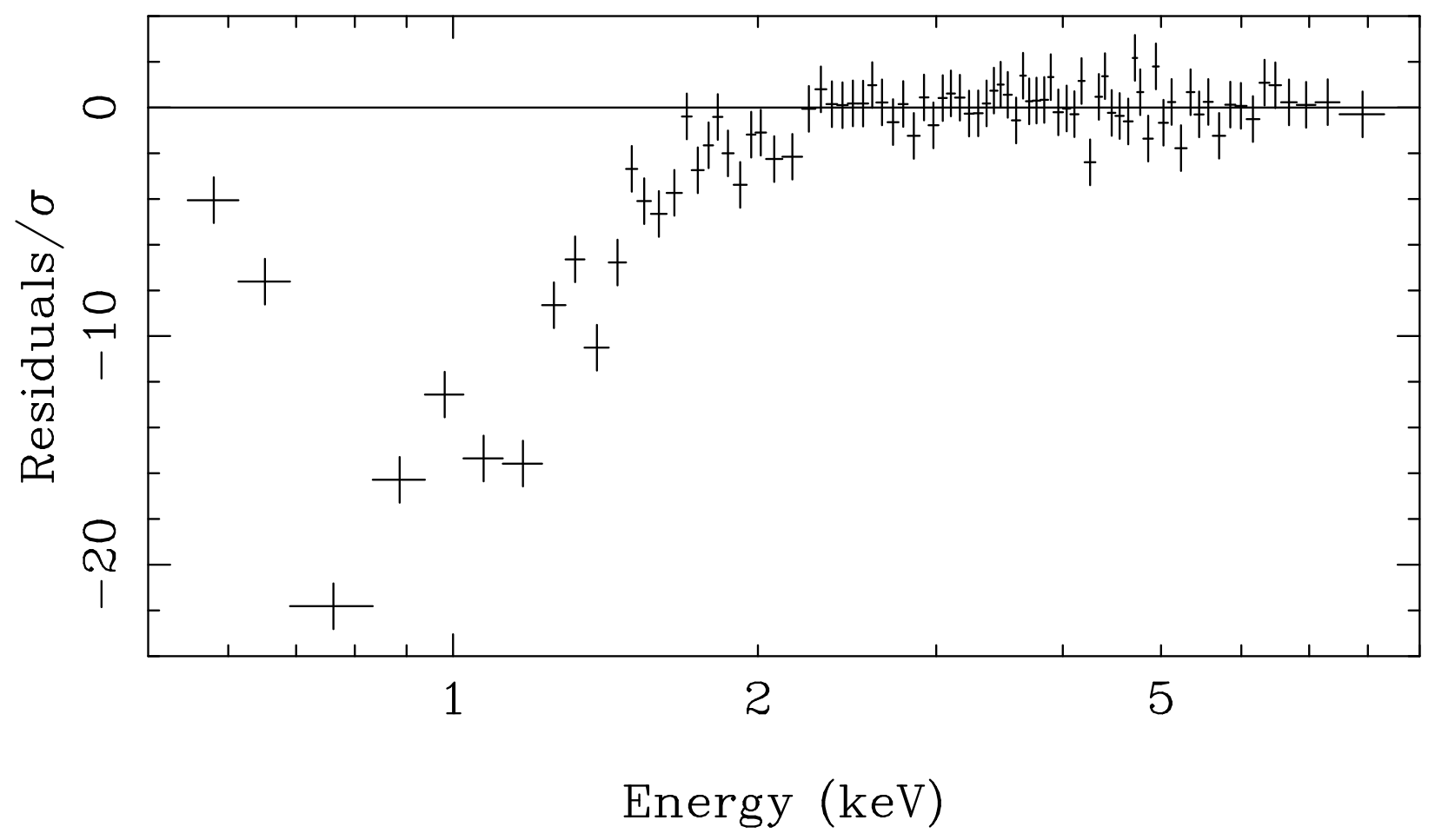

Fig. 7.- Normalized residuals from a power-law fit to the ACIS-S spectrum of NGC 4395 above $2.2 \mathrm{keV}$. The absorption has been fixed at the Galactic value. The deep troughs seen below $2 \mathrm{keV}$ suggest additional absorption by an ionized medium. 


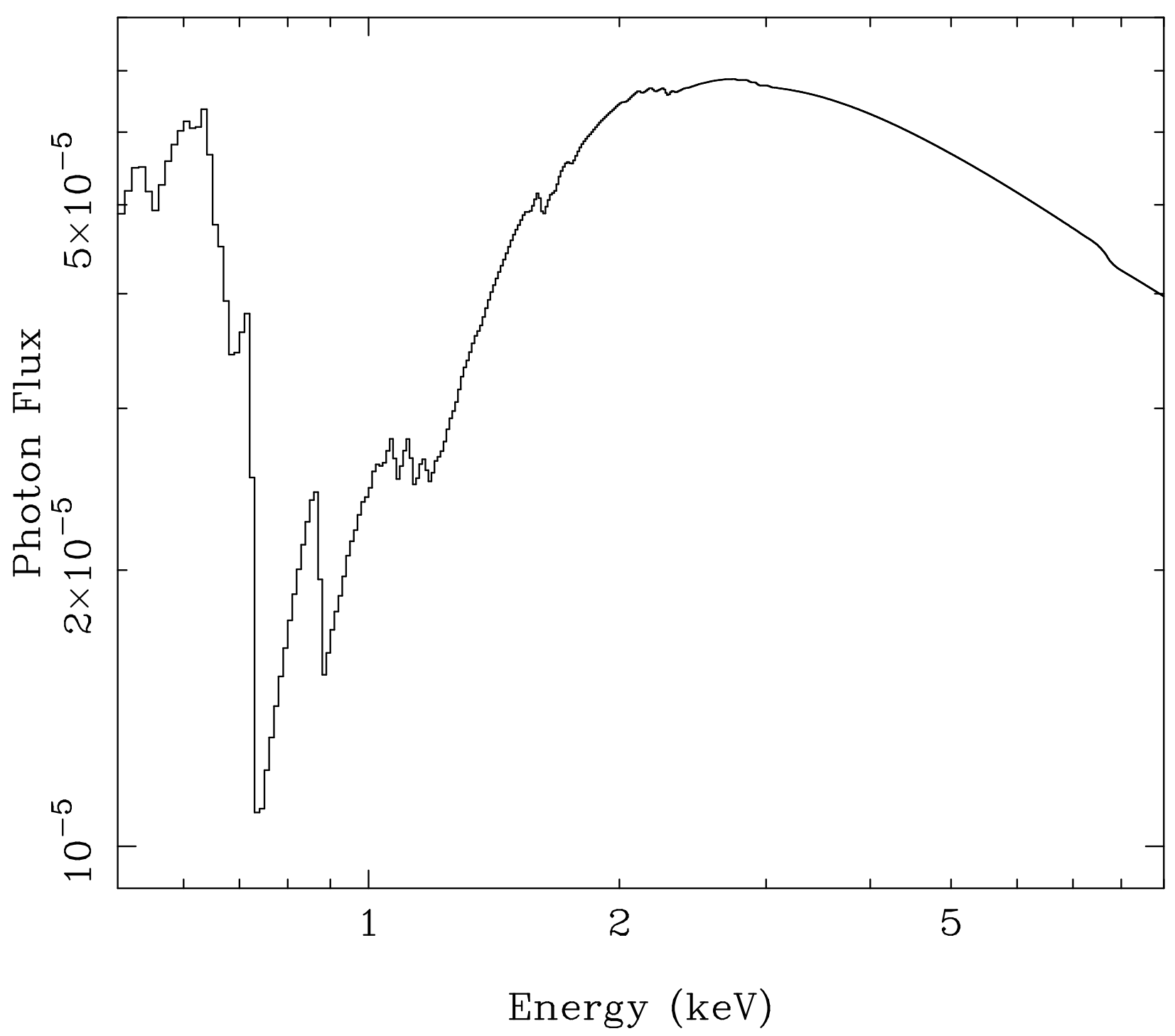

Fig. 8. - The best-fit single warm absorber model for the ACIS-S spectrum of NGC 4395. The ordinate has units of photons $\mathrm{cm}^{-2} \mathrm{~s}^{-1} \mathrm{keV}^{-1}$. 


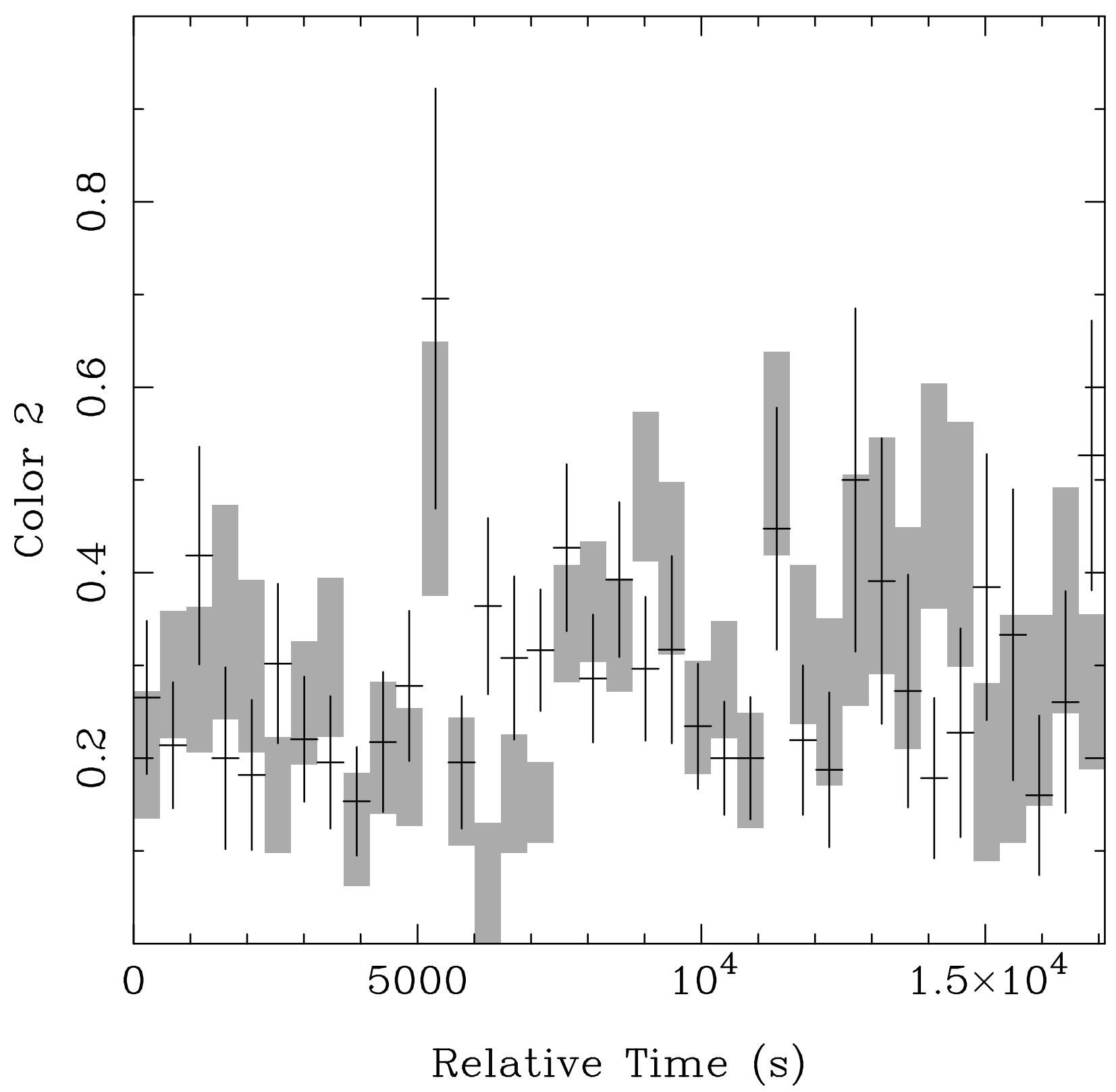

Fig. 9.- Measured (black crosses) and predicted (gray bars) values of the Color 2 parameter. The latter were derived from the observed values of Color 1 shown in Fig. 3, under the assumption that color changes arise from column density variations in the ionized medium alone. The length of the gray bars reflects the uncertainty in Color 1. 